\title{
The signals of partial restoration of chiral symmetry in medium at intermediate energy: experimental data vs. theory
}

\author{
S.M. Eliseev* \\ Bogoliubov Laboratory of Theoretical Physics \\ Joint Institute for Nuclear Research 141980 Dubna Russia
}

E-mail: selisetheor.jinr.ru

In this talk a variety of aspects of in-medium behavior of hadrons are reviewed with an emphasis on experimental data. Examples for theoretical predictions of in-medium effect from scattering and reaction processes with $K^{+}$beams below $800 \mathrm{MeV} / \mathrm{c}$ are given. It is also emphasize that final state interactions can have a famous effect on observables and thus have to be intended as part of the theory. This is demonstrated with examples from neutrino-nucleus deep inelastic interactions. In the end, the possibility to obtain hadron formation times in high-energy neutrinoinduced reactions is illustrated. The formation length parameter $L_{f}$ of pions (in the system of a moving particle) was found to be $\approx 0.5 \mathrm{fm}$.

XXI International Baldin Seminar on High Energy Physics Problems September 10-15, 2012

JINR, Dubna, Russia

${ }^{*}$ Speaker. 


\section{Introduction}

The interaction of hight and intermediate energy (IE) particles with nuclei is considered as one of the central subjects from the beginning of nuclear physics. A considerable amount of experimental data for the interaction of different strongly (as well as weakly) interacting particles from nuclei at those energies have been accumulated.

Many years ago, experiments and theory focused mainly on qualitative peculiarities of reaction mechanisms [ [U, []]. The new trends in nuclear structure investigations began after the discovery of EMC-effect and cumulative particles production [B].

Atomic nuclei, which consist of nucleons located on distances $\approx 1 \mathrm{fm}$, are excellent microlaboratories for studding elementary particles and fundamental interactions in medium. At present time, they are widely utilized for the investigation of the processes at short space-time intervals (interaction of short living particles with nucleons, fragmentation, hadronization, and that all, see

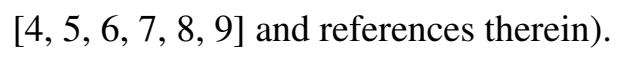

The significance of in-medium properties appeared in the 90's when some authors predicted a connection between in-medium masses and chiral symmetry restoration in hot and/or dense matter. This seemed to establish a direct link between nuclear properties on one hand and QCD property on the other. Thus hadronic models are needed for a more specific prediction of properties in medium.

However, partly due to our present lack of knowledge about the details of the confined mechanism, we are far away from a possibility to resolve the problem of in-medium effects from "the first principles". Every attempt to extract some information concerning in-medium effect from data will have (in part) the phenomenology. The authors of Ref. [ए]] (Professors Toshimitsu Yamazaki, Yoshimori Akaishi) outlined the genuine situation with the investigation of in-medium effects and highlighted the current problem : "There are a number of theories in this respect. The aim ... is to provide an interface between experimental observables and theoretical quantities".

One of the most complete information about the in-medium effects was obtained in the experiments performed by physicists at GSI. The deeply bound $\pi$ states in nuclei have been discovered and the mass shift of $\pi^{-}$in Pb nucleus was obtained. A decreased chiral order parameter, $\left(f_{\pi}^{n u c l} / f_{\pi}^{\text {free }}\right)^{2} \approx 0.78$, of deeply bound states of pions in nuclei from the experiment using a $T_{d}=604.3 \mathrm{MeV}$ at GSI, Darmstadt, was extracted [Ш], ए2].

The study of in-medium properties of hadrons has attracted quite some interest among experimentalists and theorists because of a possible connection with chiral symmetry restoration in hot and/or dense matter. Experiments using relativistic heavy ions are aimed to produce a system at very high densities and connected with that very high temperatures. In their dynamical evolution they run through various states, from an initial high-nonequilibrium stage through a very hot stage of a new state of matter (QGP). Any observed signal necessarily represents a time-integral over all these physically quite distinct states of nuclear matter. On the contrary, in experiments with microscopic probes on cold nuclei one tests interactions with nuclear matter in a well-known state, close to cold equilibrium. Even though the density probed is always smaller than the nuclear saturation density, the expected signals are as large as those from ultrarelativistic heavy-ion collisions.

In addition, there is an evidence that partial restoration can be observed even in the ground state of nucleus [ [1], [2]. (In the lowest order of density, this can be easily shown, see, T. Hatsuda, S.H. Lee, and H. Shiomi, Phys. Rev. C 52, (1995) 3364.) This situation can direct to a some 
bridge between the investigations at intermediate and hight (IE) and high energy. Experiments at IE are complimentary to heavy ion collision experiments and will give a good reference points for theories such, e.g., as the QCD sum rule. Every attempt to extract some information concerning in-medium effect from data will have (in part) the phenomenology [[13]].

\section{Space-time picture of deep inelastic lepton-nucleus collisions (cumulative nucleon production and hadron formation time)}

Atomic nuclei, which consist of nucleons located on distances $\approx 1 \mathrm{fm}$, are exelent micro-

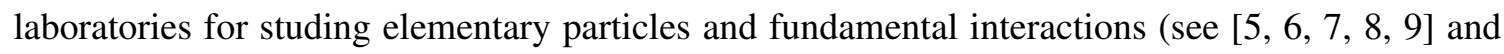
references therein). At present, the semi-inclusive hadron production in Deep Inelastic Scattering (SIDIS) of nuclear targets has became a focal point of investigations at the intersection of QCD and relativistic heavy ion physics. Its purpose is at least twofold: this reaction should help us to understand how a given QCD medium have effect on the non-perturbative hadronization mechanizms. In the second place, such a trial also can help to observe the attenuation effect or quenching of jets of partons and its formation time in nuclear medium. This is evidently a suggestive issue in the physics of high energy heavy ion collisions where the quark-gluon plasma is expected to be produced. In order to investigate the possible emergence of guark-gluon plasma, it is necessary to understand the properties of ordinary multiparticle productions mechanisms in more simple conditions than in the relativistic collisions of heavy ions [144, [5]]. In the connection with production of particles in different collisions, the concept of formation time is widely used:

from Few-Body Nuclei (see, e.g. M.A. Braun, C. Ciofi degli Atti, L.P. Kaptari, H.Morita, "Finite Formation Time in Electro-Disintegration of Few-Body Nuclei, " arXiv:nucl-th/0308069), to Heavy Nuclei:

1.) Peter Filip, Jan Pisut, "Hadron Formation Time and Dilepton Mass Spectra in Heavy Ion Collisions, " arXiv:nucl-th/9705051,

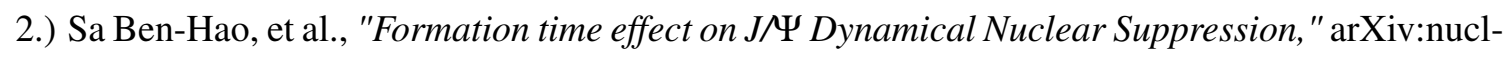
th/9803033,

Hadronization is a large distance process for which we only have models. We developed a cascade model of multiproduction of neutrino-nuclei interaction. The model describes a branching process of the evolution of parton's jet (up to hadronization) in the atomic nucleus. We assume that the interaction between incident an lepton and a target nucleus takes place in a lepton-nucleon interaction. The nucleus is excited by a series of collisions between secondaries (produced in the first lepton-nucleon interaction) and the intranuclear nucleons. This process continues until all secondaries escape target nucleus. A part of the energy is spread through the nucleus to produce a fully-equilibrated nucleus which then decays statistically (as seen in Fig.1).

The process of generation of particles is simulated by the Monte Carlo method. The characteristics of the partons from neutrino-nucleon interaction and of the produced particles with nucleons in nucleus are taken from experiments with free nucleons. (The parton spectra is assumed to be the same as hadronic one. The space-time characteristics of lepton-nucleon interactions inside the

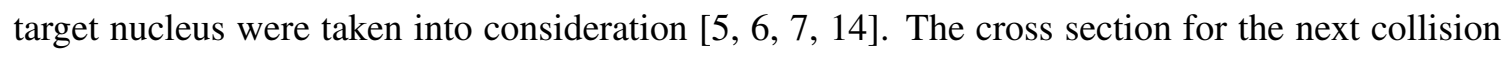
of a secondary particle with a nucleon inside the nucleus is given by

$$
\sigma_{h N}=\sigma_{h N}^{e x p}\left(1-e^{-\tau / \tau_{0}}\right),
$$


where $\tau$ is the time from the moment of production of this particle in the previous collision and $\sigma_{h N}^{e x p}$ is the experimentally determined total interaction cross section of a hadron with a nucleon in nucleus at the energy of the secondary particle produced. Thus, only after a time $\tau$ (distance $L_{f}$ ) does the cross section of intranuclear interaction reach the value $\sigma_{h N}^{e x p}$-hadronic LPM effect. The parameter $\tau_{0}$ is a certain characteristic corresponding to the formation time of the secondary generated hadron [焑].
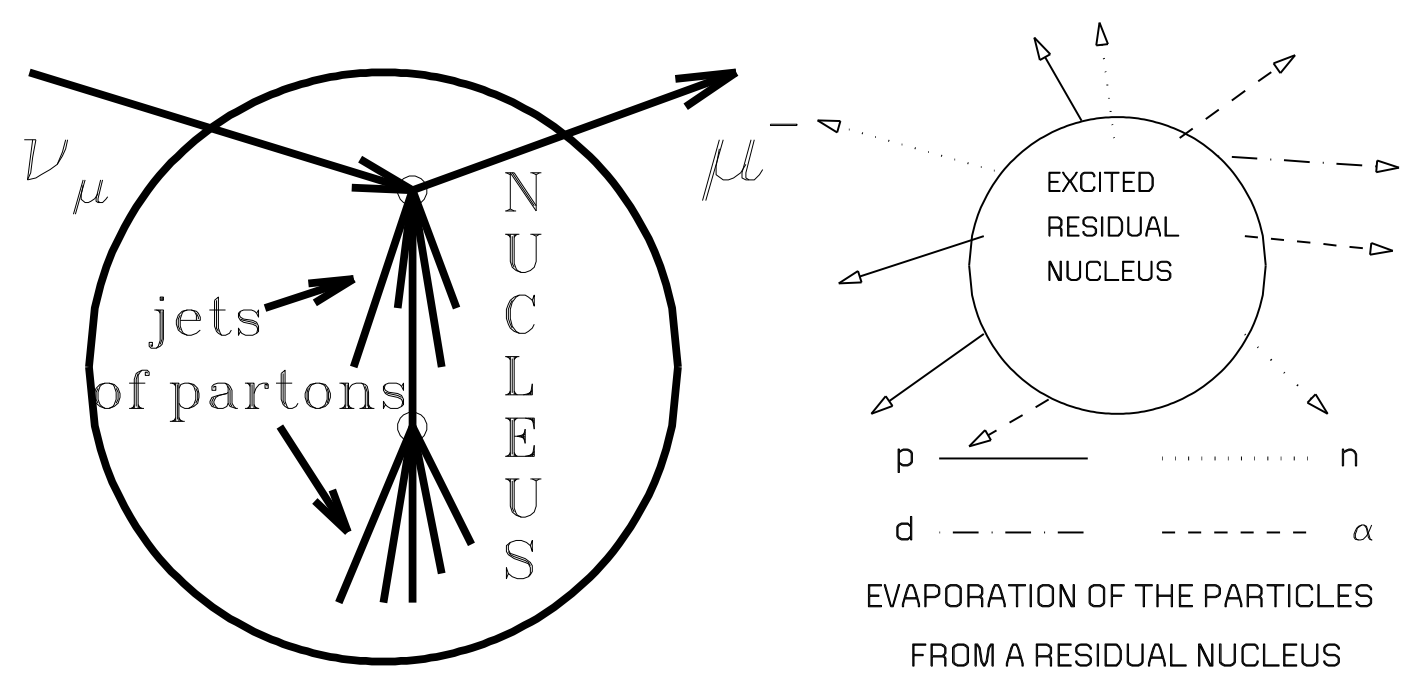

Figure 1: Left panel. Parton propagation in semi-inclusive deep inelastic scattering. Right panel. Evaporation of the particles (protons, neutrons, etc.) from exited residual nucleus.

Below, we present the theoretical results for neutrino-emulsion interaction and compare with experimental data.

Figure 2: (Left panel) shows the multiplicity distribution of relativistic s-particles for chargedcurrent $v_{\mu}$-emulsion interactions. The dotted curve corresponds to calculation according to our model with formation zone parameter $L_{f}=0.0 \mathrm{fm}$. The solid line is the prediction with $L_{f}=0.5 \mathrm{fm}$. The experimental data are taken from ref. [ $[$ ].

Figure 1:(Right panel) demonstrates the spectrum of protons emitted backwards, with respect to the beam direction, which have energies not allowed by the kinematics of collisions on a free and stationary nucleon. The doted curve manifests the of proton with $P^{2} \leq 0.2(\mathrm{GeV} / \mathrm{c})^{2}$. The mechanism of such slow proton production is a process of the evaporation of the residual nuclei excited in the stage of the propagaton of jets in the nuclei. It is worth to remark that in our approach the evolution of quark-gluon jets in nuclei in the framework of our model is accompained by a nucleon emission at backward angles and momentum $\geq 300 \mathrm{MeV} / \mathrm{c}$ (cumulative nucleons).

The spectrum of such protons is depicted by the solid curve on Figure. In our model the underlying mechanism responsible for energetic protons production was the ordinary quasideuteron intranuclear absorption process. Such Cumulative Protons( CP) were observed in deep inelastic charged-current neutrino-emulsion interactions [[1, [4]]. The experimental multillicity of CP $0.33 \pm 0.07$ are in good agreement with the calculated one equal to 0.29. The effect of intranuclear absorption of particles (pions) by intranuclear 'deuterons' is well known in the theory of nuclear reactions at intermediate energy. This process is essential only for the slow pions (of en- 

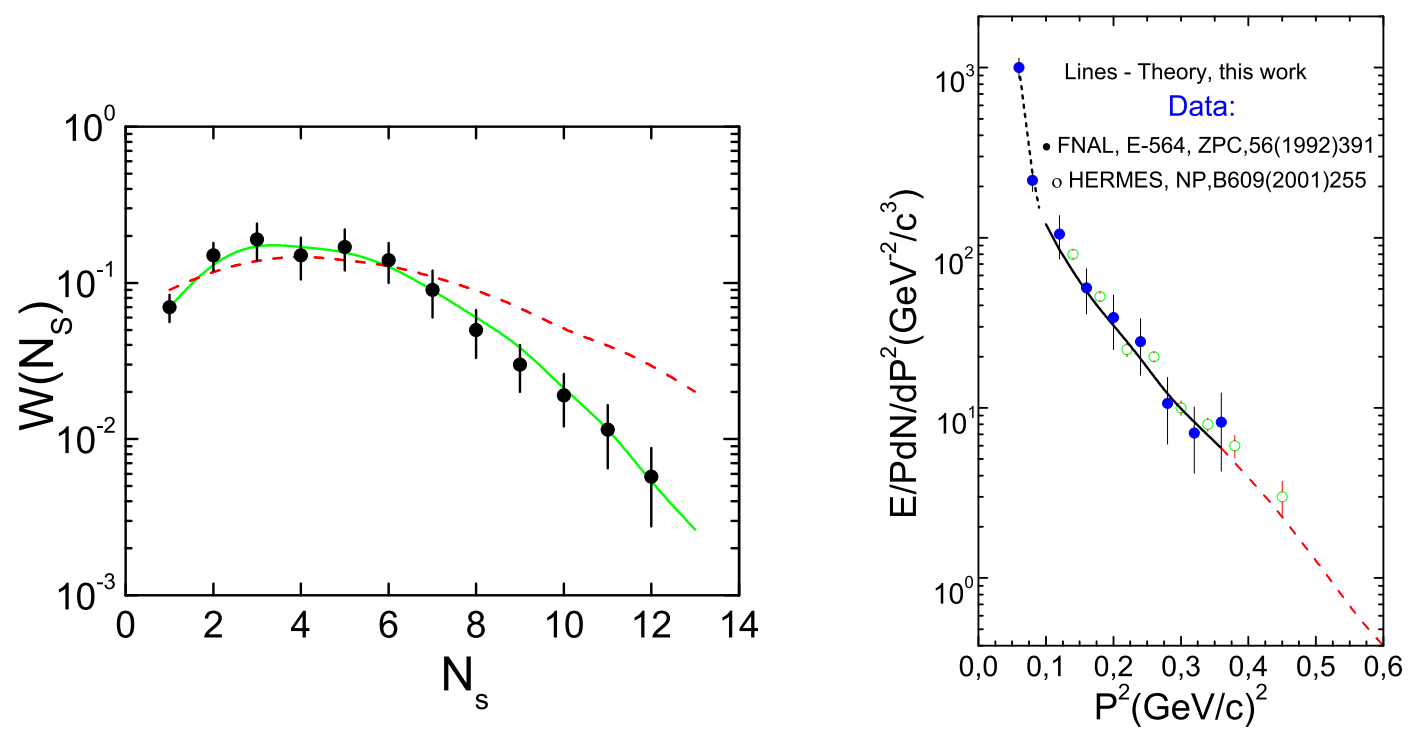

Figure 2: Left panel. The multiplicity distribution of particles for charged-current $v_{\mu}$-emulsion interactions. Right panel. Invariant momentum distributions of the final state protons from backward angles.

ergy $\leq 1 \mathrm{GeV})$. At high energy beams this effect makes all only some percents. As a result, the existing experimental data on CP from SIDIS of neutrino on nuclei can be interpreted, see solid line on Figure.

Further more, we insert the four nucleons Blokhintsev 's fluctons [ [U4]], in our model, to explore the chance to catch more energetic nucleons (than it is observed in the present day experiment. In such a way, we predicted the production of more energetic nucleons (see dashed line in Figure). This forecast can be tested in the new class of experiment with much more statistics. In addition, our estimation of zone formation is in agreement with the other finding for different particle-nucleus reactions (see $[\square, \mathbb{G}, \mathbb{\square}]$ and references therein). In conclusion, effect of the formation of particle is essential in many high energy phenomenon, e.g., in the study of signatures for QGP formation: "jet quenching", the structure of anomalous $\mathrm{J} / \psi$ suppression in nuclear collisions, etc.[15]] The new studies of the parton propagation and hadronization processes expose important connections between the DIS data, deuterium-gold collisions at RHIC, proton-nucleus interactions in Fermilab experiment E-906 at $120 \mathrm{GeV}$, and proton-nucleus collisions at the LHC with multi$\mathrm{TeV}$ beams. They are providing new tools for understanding the fundamental QCD processes (for the discussion of this subject see[Q], [6] ).

\section{3. $\mathbf{K}^{+}$-Mesons Scattering: Hints on New Effects}

Upon discovering the EMC effect (the first in-medium effect on the quark level), of great importance became the problem of detection of the signals of similar effects in nuclear reactions with hadrons. For this purpose, $\mathrm{K}^{+}-$mesons at IE are most suitable since they can probe the whole volume of nuclei owing to their small cross section of interaction with nucleons [ㅁ], [8, [1]]. 
Our basic idea is to investigate simultaneously the interactions of two various elementary particles having a like quark structure, but rather different possibilities for nuclear interactions. As follows from the experiment, the $K^{-} \mathrm{N}$ system forms a resonance at IE with a large cross section. The $K^{+}$-meson interacts with a nucleon rather weakly and, therefore can probe the full volume of nuclei to "see" some exotics in deeper levels of nuclei. (Note that $K^{-}$-mesons interact with nuclei more peripherally.)

We have used the Glauber model because it became quite standard in the theoretical physics community. She is used in various theoretical models where it is essential to calculate the propagation of particle through the medium. On the basis of this model were interpreted several experimental data, candidates for the signals of a phenomenon beyond the standard scenario: $\mathrm{J} / \Psi$ suppression and the phenomenon of color transparency (see, e.g., Refs. [155, [20]). Therefore it is very tempting to use the same theoretical approach (as an addition to the numerous calculations in the framework of usual optical model [ए], [8, [9]) to recognize whether experiment on $\mathrm{K}^{+}$-nuclei provides us with a hint for some new physics ("swelling" of the nucleon in a nuclei, renormalization of mass, i.e., partial restoration of chiral symmetry). From the theoretical point of view, this can bridging of a gap between problems of IE and high energy (relativistic nuclei collision, hadron-nuclei, QCD, QGP etc [16, [1]]). In the Glauber model, we have introduced noneikonal corrections up to thirdorder, and both Pauli and dynamic short-range correlations [22]. The nuclear Fermi motion effect was taken into account. The momenta of intranuclear nucleons are sampled by the Monte Carlo method. Relevant momentum distributions of nucleons were taken from [23]].

Following Glauber, the amplitude for a projectile-target elastic scattering, assumes the general form:

$$
f(Q)=\frac{i k}{2 \pi} \int e^{i \vec{Q} \cdot \vec{b}}\left[1-e^{i \chi(\vec{b})}\right] d \vec{b}
$$

where $b$ is the impact parameter and $\chi$ is the corresponding phase shift function. More explicitly, for projectile-nucleus scattering Eq.(3.1) can be cast into the form:

$$
F(Q)=\frac{i k}{2 \pi} \int e^{i \vec{Q} \cdot \vec{b}}<\left[1-e^{i \chi\left(\vec{b}, \vec{s}_{1}, \cdots, \vec{s}_{A}\right)}\right]>d \vec{b}
$$

where $\vec{s}_{j}$ is the component of the radius-vector $\vec{r}_{j}$ of the $j^{\text {th }}$ target-nucleon in the direction perpendicular to the incident momentum $\vec{k}$, while the brackets $<>$ denote the target ground-state average. Further, given the corresponding projectile-target nucleon amplitudes $f(q)$, one can express the above projectile-target nucleus amplitude in the parameter-free way:

$$
\begin{gathered}
F(Q)=i k) \int b d b J_{0}(Q b) \times\left\{1-\left[1-\frac{1}{2 \pi i k} \int e^{-i \vec{q} \cdot \vec{b}} f_{p}(q) S_{p}(q) d \vec{q}\right]^{Z}\right. \\
\left.\times\left[1-\frac{1}{2 \pi i k} \int e^{-i \vec{q} \cdot \vec{b}} f_{n}(q) S_{n}(q) d \vec{q}\right]^{N}\right\}
\end{gathered}
$$


here $S(q)$ is the nuclear form factor, $N, Z$ are the numbers of neutrons and protons in the target nucleus, while $G(Q)$ is the corresponding c.m. correlation factor. It is then a straightforward matter to determine the total cross section for the case of $\mathrm{K}^{+}$-target nucleus scattering according to the optical theorem. The total and reaction cross sections may then be approximated by:

$$
\sigma_{t}=4 \pi \int_{0}^{\infty} \operatorname{Re}\left[1-e^{i \chi(b)}\right] b d b, \quad \sigma_{r}=2 \pi \int_{0}^{\infty}\left[1-e^{-2 \operatorname{Im} \chi(b)}\right] b d b
$$

The parameters of kaon-nucleon amplitudes was taken from the data and from the Martin phase shifts [24, 205, [26, 227]. Having thus the amplitudes $f(I)$ for isospins $I=0$ and $I=1$ we can readily find the $\mathrm{K}^{+}$-proton amplitude as well as $\mathrm{K}^{+}$-neutron amplitude.

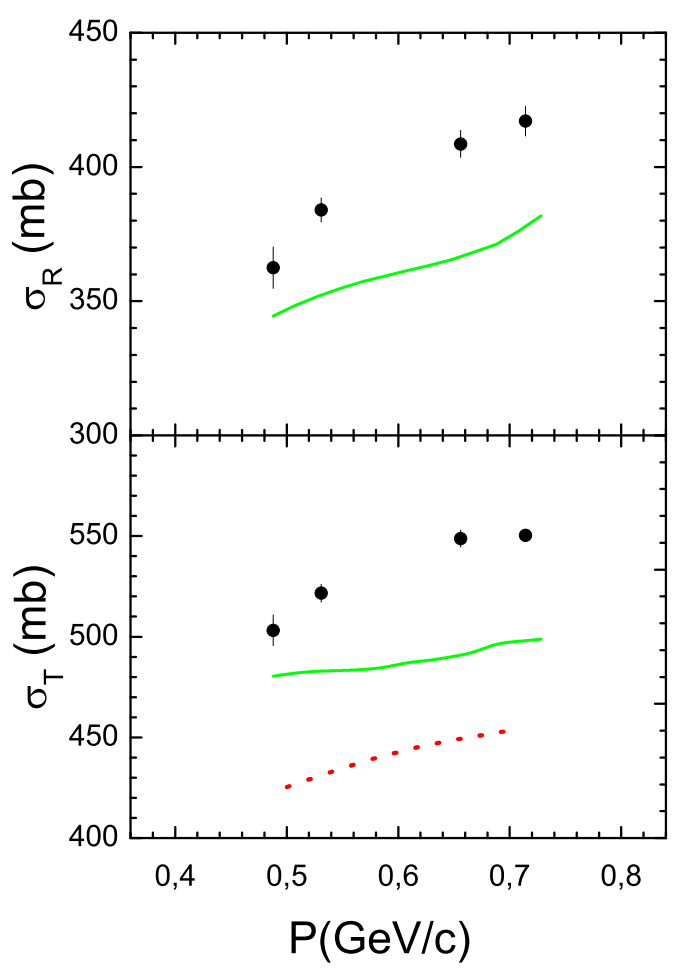

Figure 3: The total, $\sigma_{t}$, and reaction, $\sigma_{r}$, cross sections for $\mathrm{K}^{+}$-mesons interaction with calcium nuclei. The solid lines denote the prediction of our model. The dotted curve represents the result of the previous authors [28]. The experimental data are taken from Refs. [ए]]. the agreement between theory and the new experiment for the $K^{+}$-scattering (see Fig. 3). We show that the greatest puzzle is that the $K^{-}$-nuclei data are rather well described but the predictions for $K^{+}$are quenched with respect to the new experimental data. We should emphasis that our results $\mathrm{K}^{+}$meson interactions with nuclei fail to describe the experimental data. Comparison of the experimental data with the results of our calculations clear demonstrates that the deeply penetrating hadron $\left(\mathrm{K}^{+}-\right.$ meson) can "see" in the nucleus some "exotics" (beyond habitual mechanism of nuclear reactions). At the same time, our model give an adequate description of collisions of $\mathrm{K}^{-}$with targets, i.e. in the case of more peripheral interactions

(see Tab. 1) . It worth noticing that our results have been obtained without fitting any new parameters. Many authors assume some exotic phenomena ranging from the nucleon swelling to renolmalization of nucleon in a nucleus (partial restoration of chiral symmetry). Our results show the possibility of observing some unusual phenomena in $\mathrm{K}^{+}$-nucleus interaction.

The influence of all our corrections (introduced in usual Glauber model) on the calculation cross of sections of $\mathrm{K}^{-}$ and $K^{+}$-nuclei interactions is shown in Tab.1. In comparison with the results of other authors our present model improves

Fig. 3 represents our results. We can see that our calculated cross sections for 
only argue in favor of the "window" for new physics scenario in $\mathrm{K}^{+}$- nuclei collisions. Much work to be done to fully settle the nature of the $\mathrm{K}^{+}$-nucleus anomaly.

Table 1. The total cross sections (in $\mathrm{mb}$ ) for K-mesons interaction with ${ }^{12} \mathrm{C}$ nucleus at momentum $800 \mathrm{MeV} / \mathrm{c}$. In parentheses are given the value of cross sections taken from Glauber model without corrections.

\begin{tabular}{ccccccc}
\hline \multicolumn{3}{c}{$\sigma\left(\mathrm{K}^{-}+\mathrm{C}\right)$} & & & \multicolumn{3}{c}{$\sigma\left(\mathrm{K}^{+}+\mathrm{C}\right)$} \\
\cline { 1 - 3 } \cline { 5 - 6 } Data $^{a}$ & theory $^{a}$ & this work & & Data $^{a}$ & theory $^{a}$ & this work \\
\hline $338 \pm 7$ & 328 & $335(325)$ & & $177 \pm 1.5$ & 149 & $164(161)$ \\
\hline
\end{tabular}

${ }^{a}$ as in [ए]]

\section{References}

[1] N. Metropolis et al., Phys. Rev. 110, 2619 (1952).

[2] S.M. Eliseev, J.M. Kohli, Nucl.Phys. 59, 128 (1973).

[3] A.M. Baldin et al., Sov.J.Nucl.Phys. 18, 41 (1974).

[4] L.D. Landau et al., Dok. Akad. Nauk SSSR 92, 535 (1953).

[5] A. El-Naghy et al., J. Phys. G: Nucl. Part. Phys. 16, 39 (1990).

[6] N.N. Nikolaev, Usp. Fiz. Nauk 134, 369 (1980).

[7] S.M. Eliseev et al., Sov. J. Nucl. Phys. 40, 601 (1984).

[8] "Experimental Studies of Hadronization and Parton Propagation in the Space-Time Domain," W. K. Brooks and H. Hakobyan, Phys. A 830, 361c (2009).

[9] "Parton Propagation and Fragmentation in QCD Matter," A. Accardi et al., Riv. Nuovo Cim. 32, 439 (2010).

[10] Toshimitsu Yamazaki, Yoshimori Akaishi, Phys. Lett. B418, 246 (1998); Phys. Lett. B453,1 (1999).

[11] "Precision Spectroscopy of Pionic 1s States of Sn Nuclei and Evidence for Partial Restoration of Chiral Symmetry in the Nuclear Medium," K. Suzuki et al., Phys. Rev. Lett. 92, 072302 (2004).

[12] "Deeply bound pionic states in heavy nuclei," Toshimitsu Yamazaki et al., Phys. Rept. 514, 1 (2012).

[13] "The effect of the in-medium pentaquark on the kaon optical potential," Laura Tolos, Phys. Lett. B 632, 219 (2006).

[14] "Production of Cumulative Protons in DIS of Nuclei, the Role of Fluctons, LPM Effect and all that," S.M. Eliseev, Proc. of the XVII Intern. Baldin Seminar on High Energy Physics Problems "Relativistic Nuclear Physics \& Quantum Chromodynamics, "JINR, Dubna, 2005, eds. A.N. Sissakian, V.V. Burov, A.I. Malakhov, V. I, p. 84.

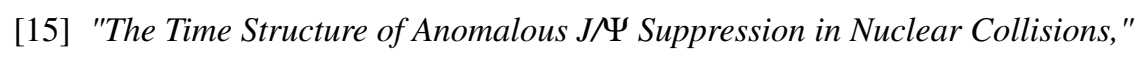
Jorg Hüfner, Pengfei Zhuang,"Proc. of XIIth International Conference on Selected Problems of Modern Physics Dedicated to the 95th anniversary of the birth of D.I. Blokhintsev (1908-1979)," Dubna, June 8 - 11, 2003, D1,2-2003-219, p.282.

[16] "New experimental tools for exploring in-medium parton propagation in $Q C D$, "William Brooks1, Hayk Hakobyan, and Miguel Arratia, PoS QNP2012_21. 
[17] E. Friedman, A. Gal, Phys. Rept. 452, 89 (2007) and references therein.

[18] D. Marlow et al., Phys. Rev. C 25, 2619 (1982).

[19] G.E. Brown, C.B. Dover, P.B. Siegel, and W.Weise, Phys. Rev. Lett. 60, 2723 (1988).

[20] A.S. Carrol et al., Phys. Rev. Lett. 61, 1698 (1988) 1698.

[21] M. Asakawa, Nucl. Phys. A 629, 344c (1998).

[22] S.J. Wallace, J.L. Friar, Phys. Rev. C29, 956 (1984).

[23] A.N. Antonov et al., Phys.Rev. C74, 054603(2006).

[24] K. Hashimonto, Phys. Rev. C 29, 1377 (1984).

[25] J.S. Hyshop et al., Phys. Rev. D 46, 961 (1992).

[26] B.R. Martin, Nucl. Phys. B 94, 413 (1975).

[27] B.R. Martin,R. Perrin, Nucl. Phys. B 20, 287 (1970).

[28] M.F. Jiang et al., Phys. Rev. C 51, 857 (1995). 